(C) 2019 International Journal of Nursing and Health Services

This is an Open Access article distributed under the terms of the Creative Commons Attribution 4.0 International License.

REVIEW ARTICLE

\title{
PERSONALIZED COGNITIVE COUNSELING IN PREVENTING RISK BEHAVIOR AMONG MEN WHO HAVE SEX WITH MEN (MSM): A LITERATURE REVIEW
}

\section{Lussy Afriyanti ${ }^{*}$, Agung Waluyo²}

\author{
1 Master Student of Medical and Surgical Nursing, Faculty of Nursing, Universitas \\ Indonesia, Depok, Indonesia \\ 2 Department of Medical and Surgical Nursing, Faculty of Nursing, Universitas Indonesia, \\ Depok, Indonesia \\ * Correspondence: lussyafriyanti83@gmail.com
}

\begin{abstract}
The current trend of HIV infection shows an increase in cases among men who have sex with men (MSM). Risk behavior in MSM has shown a high prevalence of moderate and worrying risk behaviors for HIV infection, including unprotected anal intercourse (UAI) and drug use. This literature review aims to explore the effectiveness of Personalized Cognitive Counseling (PCC) interventions in reducing risk behaviors for HIV transmission to MSM. Articles discussed in this literature study were obtained from several databases using the keywords "personalized cognitive counseling," "men who have sex with men," and "risk behavior." Cognitive counseling interventions are effective in reducing episodes of anal intercourse. Adapted Personalized Cognitive Counseling (PCC) intervention also has been effective in reducing sexual risk behavior in drug users MSM. This intervention is possible and essential to developing in dealing with risk behavior in MSM that tends to increase in its population.
\end{abstract}

Keywords: cognitive counseling, personalized cognitive counseling, men who have sex with men (MSM), risk behavior

International Journal of Nursing and Health Services (IJNHS), September 2019, Volume 2, Issue 3; Page 32-38

Received: 02 April 2019; Revised: 22 April 2019; Accepted: 04 May 2019

Doi: http//doi.org/10.35654/ijnhs.v2i3.114

\section{Introduction}

The epidemic of Human Immunodeficiency Virus (HIV) infection is a global health problem that is very complicated to date. According to UNAIDS, in 2016, 36.7 million people were living with HIV/AIDS spread in the world, with 1.8 million new infections and 1 million deaths (1). While in 2017, there were 36.9 million people in the world lived with HIV/AIDS, 1.8 million new infections, and 940,000 people died from AIDS (2). At present, the trend of HIV infection shows new infections among men who have sex with men (MSM). Based on the behavior risk analysis of MSM, it showed only $26.0 \%$ of MSM who were at lower risk, $54.2 \%$ at moderate risk, and $20.0 \%$ at very high risk of HIV (3). The term MSM, 
such gay men who are culturally specific and personally meaningful as choices in describing their feelings, romantic attractions, and sexual behavior (4).

The behaviors of MSM who are associated with HIV transmission are sexual risk intercourse and drug use both before and during sexual activities. The drugs used have a variety of effects ranging from the libido, self-confidence at sexual activities, feelings of social relations and immunity, hope of cognitive escape and sexual satisfaction, and assessment disorder. These effects have implications as psychological factors that cause MSM to take risks on higher sexual activities while under the influence of substances (5).

Cognitive behavior counseling interventions that are accompanied by HIV testing have proven effective in reducing episodes of anal intercourse in serodiscordant couples for HIV negative MSM (6-7). Based on the literature presented above, the author interested in discussing some of the research about cognitive counseling issued to MSM in the relation of interventions to prevent risk behavior which can cause in transmitting HIV infection between MSM community.

\section{Objectives}

This literature study aims to explore the effectiveness of personalized cognitive counseling intervention in reducing risk behavior for HIV transmission in MSM.

\section{Methods}

The articles discussed in this literature study from several databases, including MEDLINE, CINAHL, Scopus, and Proquest. The keywords were used, including "personalized cognitive counseling," "men who have sex with men" and "risk behavior." This study limits the number of articles from 2007 to 2018, and we have obtained 9 (nine) relevant articles for this literature study.

\section{Results}

\subsection{Description of studies}

Seventy-two related articles were selected in this study. However, only nine full-text articles that matched the criteria following the literature review. Once the inclusion criteria were applied, we excluded 63 articles because not relevant to the objectives. About 63 articles were banned, such as studies that concerned men who have sex with men. Thirtytwo articles out of from the theme. Thirteen articles do not focus on MSM, eleven articles were not focused on risk behavior, and seven duplicate articles. Nine most relevant articles extracted including two quasi-experiment articles, five randomized controlled trials (RCT) articles, one qualitative study article, and one systematic review article.

\subsection{Definition of Personalized Cognitive Counselling (PCC)}

The PCC is a transmission counseling intervention to reduce cognitive risk reduction. It was sufficient for reducing sexual intercourse at high risk of HIV transmission. Two randomized controlled trials by Dilley et al. in 2002, and 2007 conducted on HIV negative men at high risk of having sex with men (MSM) and have repeatedly carried out HIV tests (6).

According to the cognitive psychology hypothesis, Ron Gold and his colleagues, PCC interventions can be defined as the decision to have high-risk sexual relations by minimizing known risks (6). However, many MSMs take the risk by engaging in sexual 
behavior even though they know the potential for HIV infection (8). The PCC facilitated patients who have not been involved in unprotected Anal Intercourse (UAI) could rationalize their decisions when sexual arousal arises (7).

\subsection{PCC Intervention}

According to the CDC (2018), PCC can be defined as the intervention that focused on a single counseling session and included risk assessment pre-test and post-test to find out the results of counseling. During the intervention session, the counselor asked the client to remember the latest unprotected anal intercourse with a man, and then they also asked to describe what kind of sexual activities. Also, counselors and clients identified and shared the feelings and experience that lead to high-risk sexual behavior. Thereby, they can decide to choose the best strategies future (9).

In the initial research conducted by Dilley et al., PCC interventions were delivered in the context of individual session HIV testing counseling for 30 to 50 minutes (10). The arrangement of response was carried out at the community HIV testing clinic. Counselors are trained first in counseling and HIV prevention tests and have at least one year of experience doing HIV counseling. The research process was assisted by the PCC questionnaire, a self-justification list that MSM often uses to rationalize risky behaviors. PCC intervention consisted of five steps, including, 1) helping patients to remember the impressive episodes from UAI; 2) filling out the PCC questionnaire based on the specific event experienced, discussion with patients about the event, along with the thoughts and feelings suffered by the client; 3) helping patients to identify self-justification; 4) and talk about avoiding risky sexual behaviors the similar situation in the future to $(8,11)$.

\subsubsection{Personalized Cognitive Counseling in Preventing Sexual Risk Behavior}

Two PCC controlled studies conducted at the AIDS Health Project (AHP) in San Francisco by Dilley et al. in 2002 and 2007. In the first study, 248 MSM were eligible for PCC intervention. Two intervention groups received standard HIV test counseling plus PCC cognitive behavior intervention, carried out by mental health professionals, and two control groups only received standard HIV test counseling (11). A similar study in a second study which testing PCC versus standard HIV testing counseling using experienced undergraduate HIV test counselors trained to provide PCC interventions. Participants in the second study were 336 randomized MSM who received PCC intervention or HIV counseling $(10,11)$. Those studies showed that PCC intervention significantly reduced the number of UAI episodes with non-permanent partners who had HIV-positive status or unknown HIV status within six months after counseling. In the first study, the average number of incidents of risky sexual intercourse significantly decreased by about half at six months of follow-up and could be maintained 12 months later (11).

The PCC interventions also have proven effective for all racial groups of both white and colored skin after six months of intervention and lower in glowing skin after 12 months of intervention. Among white and people of color, a decrease in UAI episodes of unprotected anal intercourse in PCC participants occurred at six months relative to standard management (6).

While according to research by Schwarcz et al., there was weak and indirect evidence for the potential effect of PCC in reducing sexual risk behavior in MSM populations. A more significant reduction in the number of average UAI episodes in the PCC 
group, especially at 12 months, but the difference was not statistically significant at both time points for the great effect of PCC treatment. The decrease in UAI reports was slightly more significant in the control group, and no differences observed in the incidence of sexually transmitted infections (STIs) (7). The addition of a second "booster" session, which is six months after initial counseling may be needed to achieve a higher and more lasting risk reduction (6). Also, most MSM who received PCC intervention earlier tended to report that counseling could reduce the likelihood that they would be involved in the next UAI epidemic (7).

\subsubsection{Personalized Cognitive Counseling for MSM Episodic Drug Users}

PCC associated with reduced risk of HIV transmission to MSM through risky sexual intercourse. They required to involve in vital component including self Justification Elicitation Instrument (SJEI), which is a positive effect on engaging to the unprotected anal sex with the use of drugs simultaneously (5).

A study of a randomized controlled trial conducted by Coffin et al. found that the PCC intervention has positive effects on reducing sexual risk behavior (8). Another study also mentioned that the cognitive-behavioral intervention was a significant decrease in the number of unprotected anal intercourse after 12 months of intervention (12).

Therefore, the

PCC has benefits for MSM drug users in filtering drug use, reducing the number of UAI events and the number of UAI events with the most recent non-permanent partners (8). Because according to a qualitative study by Knight et al. (2014) have shown specific reasons engaging in high-risk behavior resonating with MSM in general. The reasons that arose from the qualitative analysis include the search for sensations or spontaneity, problems related to condoms, opportunities for sexual intercourse, and expectations or obligations towards a partner (5).

PCC adapted for episodic drug users MSM, involving short counseling sessions with rapid HIV testing. However, PCC intervention on MSM who experience drug dependence cannot be expected to affect sexual risk behavior among MSM who are dependent on drugs. However, there was substantial evidence reported there is a relationship between the frequency of drug use and the risk of HIV transmission(8). Therefore, MSM has symptoms of drug dependence should be directed to more intensive services.

\section{Discussion}

Men who have sex with men (MSM) are currently a high-risk group in HIV transmission $(3,13)$. Risk behavior that is a trend in the LSL community has become a factor that causes this community to have a greater tendency to experience sexual infections, including HIV. Risk behaviors, especially unprotected anal intercourse, even those accompanied by substance use, show higher numbers among MSM communities than the general population (13). In this review discussed interventions to overcome risk behavior in MSM, which is personalized cognitive counseling.

Personalized Cognitive Counseling (PCC) is a counseling intervention to reduce high-risk behaviors among men who have sex with men (MSM) $(6,11)$. In the PCC intervention, patients were required to decide some best strategies when faced problem related to the high-risk sex (11). PCC known as the REP Replicating Effective Programs (REP) supported by the translation of evidence-based HIV/AIDS prevention interventions. 
The intervention has been field-tested in two community agencies by non-research staff (9). PCC is for HIV-seronegative MSM, who have had at least one HIV test, and who report unprotected anal sex (UAI) in the last 12 months with an unknown HIV serostatus partner or an HIV-positive partner. PCC is delivered in the context of HIV testing counseling in individual sessions 30 to 50 minutes (11).

This intervention was used in the initial research conducted by Dilley et al. in 2002 with 248 participants. Two intervention groups received standard HIV test counseling plus the PCC cognitive-behavioral intervention, delivered by mental health professionals, and two control groups received only standard HIV test counseling (11). A second similar study in 2007 tested PCC versus standard HIV testing counseling with 336 MSM as participants who were randomly selected to be given standard PCC intervention or HIV counseling (10). In those studies, showed that PCC intervention significantly reduced the number of UAI episodes in MSM with non-permanent partners HIV-positive or unknown status partners in six months after intervention $(10,11)$.

Another RCT study conducted by Dilley et al. (2011) in proving the effectiveness of PCC interventions in men of colored (MOC) and whites also showed significant results in both groups of participants. Among MOC and whites, the decline in episodes of UAI among PCC participants occurred at six months relative to usual counseling (UC). It may represent the lowest risk level that can be achieved with a single counseling session in this group of high-risk men. It is may be necessary to add a second 'booster' session six months after the initial counseling to achieve a more significant and more durable decline in risk (6).

Another study conducted by Schwarcz et al. (2013) showed 374 HIV-positive MSM after receiving two sessions of PCC intervention was significantly different than the control group. After six months of response, the number of UAI episodes decreased in two groups and declined further in the PCC group after 12 months of intervention. The findings from this randomized trial do not support the efficacy of a two-session PCC intervention at reducing UAI among HIV-infected MSM and indicate the continued need to identify and implement effective prevention methods in this population (7). However, there is a positive effect of risk behaviors among the intervention group who received the cognitivebehavioral intervention than the control group who received the standard care. Therefore, multilevel interventions are needed to provide preferential results compared to control groups; interventions that focus on more structural and policy-level interventions could provide broader and more impactful behavior change (12).

MSM reported a variety of high-risk activities including not using condoms for anal sex, having sex while under the influence of alcohol or drugs (14-15), crystal methamphetamine use as a significant driver behind high-risk sexual behavior among MSM (16). PCC that was adapted and provided with other interventions had a positive impact on MSM with the risk of substance use. Adapted PCC can benefit MSM drug users in filtering drug use so they will not experience substance dependence, and among MSM who were not dependent, there was a statistically significant reduction in the total number of UAI events and the number of UAI events with the most recent non-permanent partners (8). Adding behavioral activation to risk reduction counseling for MSM with problematic crystal methamphetamine use may augment the potency of a risk reduction intervention for this population (15).

However, A previous study showed there is no significant effect on reducing sexual risk behaviors (8). In this case, an intervention with a combination approach is needed in 
modifying high-risk behavior in episodic substance-use MSM, especially when they are under the influence of drugs and alcohol.

The results of this review may not be generalized for other intervention modalities since we have a limit of studies. Although some studies showed there was a positive effect on outcomes but not much different from the control group that received standard counseling.

We have a limited assessment of the effectiveness of personalized cognitive counseling, implementation, and level of excellence. However, this PCC can support the evidence of HIV/AIDS based prevention program for implementing in the clinical setting.

\section{Conclusion}

This literature study has been supported by previous studies that provide support for reducing sexually transmitted diseases through unprotected anal intercourse. Interventions of Personalized Cognitive Counseling (PCC) effectively in reducing sexual risk activities among MSM with non-permanent partner HIV-positive and unknown status partner. Adapted PCC is also useful for MSM drug users who do not have drug dependence to reduce their risk behavior. Because of the increase of HIV epidemic and the trend of HIV prevalence in the world, there is an urgent need to develop and to support interventions related to various aspects regarding the risk behavior of MSM, namely with Personalized Cognitive Counseling (PCC).

\section{References}

1. Joint United Nations Programme on HIV and AIDS (UNAIDS). Data. 2017. http://www.unaids.org/en/resources/documents/2017/2017_data_book

2. Joint United Nations Programme on HIV and AIDS (UNAIDS). 2017 Global HIV $\begin{array}{ll}\text { statistics. } & 2018 .\end{array}$ http://www.unaids.org/sites/default/files/media_asset/UNAIDS_FactSheet_en.pdf

3. Rocha GM, Kerr SLRF, Kendall C, Guimarães MDC. Risk behavior score : a practical approach for assessing risk among men who have sex with men in Brazil. Brazillian Journal of infectious diseases. 2018;2(2):113-122.

4. World Health Organization (WHO). HIV and other sexual health considerations for young men who have sex with men. 2018. https://www.who.int/hiv/pub/populations/out-with-it/en/

5. Knight KR, Das M, Demicco E, Antunez E, Santos G, Dadasovich R. A roadmap for adapting an evidence-based HIV prevention intervention: personal cognitive counseling ( PCC ) for episodic substance-using men who have sex with men. Prevention of science. 2014;15:364-375. https://doi.org/10.1007/s11121-0130364-z

6. Dilley JW, Schwarcz S, Murphy J, Joseph C, Vittinghoff E, Scheer S. Efficacy of personalized cognitive counseling in men of color who have sex with men: secondary data analysis from a controlled intervention trial. AIDS behavior. 2011;15:970-975. https://doi.org/10.1007/s10461-010-9771-3

7. Schwarcz SK, Chen Y-H, Murphy JL, Paul JP, Skinta MD, Scheer S, et al. A randomized control trial of personalized cognitive counseling to reduce sexual risk among HIV- 
infected men who have sex with men. AIDS Care. 2013;25(1):1-10. https://doi.org/10.1080/09540121.2012.674095

8. Coffin PO, Santos G, Colfax G, Das M, Matheson T, DeMicco E, et al. Adapted personalized cognitive counseling for episodic substance-using men who have sex with men: a randomized controlled trial. AIDS behavior. 2014;18:1390-1400. https://doi.org/10.1007/s10461-014-0712-4

9. Centers for Disease Control and Prevention (CDC). Risk Reduction (RR) ChapterPersonalized Cognitive Counseling. 2018. https://www.cdc.gov/hiv/pdf/research/interventionresearch/compendium/cdchiv-personalized_cog_counseling_pcc_best_rr.pdf

10. Dilley JW, Woods WJ, Loeb L, Nelson K, Sheon N, Mullan J, et al. Brief cognitive counseling with HIV testing to reduce sexual risk among men who have sex with men. Journal of acquired immune deficiency syndromes. 2007;44(5):569-577.

11. Centers for Disease Control and Prevention (CDC). Personalized Cognitive Counseling (PCC) A Single Session Intervention for MSM Who Are Repeat Testers for HIV. 2015. https://www.cdc.gov/hiv/research/interventionresearch/rep/packages/pcc.html

12. Mansergh G, Koblin BA, Mckirnan DJ, Hudson SM, Flores SA, Wiegand RE, et al. An intervention to reduce HIV risk behavior of substance-using men who have sex with men: a two-group randomized trial with a nonrandomized third group. Plos medicine. 2010;7(8):1-10.

13. Melendez-torres GJ, Bonell C. Systematic review of cognitive-behavioral interventions for HIV risk reduction in substance-using men who have sex with men. International Journal of STD \& AIDS. 2014;25(9):627-35.

14. Williams PP, Carney T, Plüddemann A, Parry CDH. Intervening to identify and reduce drug use and sexual HIV risk patterns among men who have sex with men in three provinces in South Africa. Journal of substance use. 2014;19(1-2):141-146.

15. Khumaidi, Yona S. Determinants factors of condom use among men sex men and transgender women in the Southeast Asia region: A literature review. International Journal of Nursing and Health Services. 2018; 1(2):10-18

16. Mimiaga MJ, Reisner SL, Pantalone DW, O'Cleirigh C, Mayer KH, Safren SA. A pilot trial of integrated behavioral activation and sexual risk reduction counseling for HIVuninfected men who have sex with men abusing crystal methamphetamine. AIDS patient care and STDs. 2012;26(11). 